\title{
A COMPARISON OF DIESEL SPRAYS CFD MODELING APPROACHES: DDM VERSUS $\Sigma$-Y EULERIAN ATOMIZATION MODEL
}

\author{
J. M. Desantes, J. M. García-Oliver, J. M. Pastor, ${ }^{*} \mathcal{E}$ \\ A. Pandal
}

CMT-Motores Térmicos, Universitat Politècnica de València, 46022, Spain

*Address all correspondence to: J. M. Pastor, E-mail: jopasen@mot.upv.es

Original Manuscript Submitted: 01/09/2015; Final Draft Received: 05/12/2015

\begin{abstract}
A comparison between the $\Sigma-Y$ atomization model and a classical DDM approach has been carried out for diesel spray computational fluid dynamics (CFD) simulations. The $\Sigma-Y$ model, originally proposed by Vallet and Borghi, is based on a Eulerian representation of the spray atomization and dispersion by means of a single-fluid variable density turbulent flow. The locally homogeneous flow approach has been applied to develop a spray vaporization model based on state relationships. A finite-volume solver for model equations has been created using the OpenFOAM CFD open-source $\mathrm{C}++$ library. In the case of the Lagrangian-discrete droplet method (DDM) approach, the original dieselFoam solver of OpenFOAM is used. Model predictions have been compared to experimental measurements of free diesel sprays under vaporizing conditions from the database of the Engine Combustion Network (ECN). Accurate predictions of liquid and vapor spray penetration, as well as mixture fraction, can be achieved for the nominal condition with both models, although DDM simulations tend to be less accurate. Additionally, the near nozzle flow structure of the Spray A condition of ECN is also studied with both models. The conclusion is a more accurate prediction of the near-field internal structure of the spray in the case of the Eulerian model, due to both a higher mesh resolution and a more adequate modeling approach. Consequently, results shown in this work put in evidence the benefits of using a Eulerian model to predict qualitatively and accurately the diesel spray behavior under different ambient conditions and injection pressures.
\end{abstract}

KEY WORDS: Eulerian, Lagrangian, diesel, evaporation, CFD, OpenFOAM ${ }^{\circledR}$

\section{INTRODUCTION}

Regulations on diesel engine pollutant emissions have become more restrictive in the last years all over the world and specially in the European Union. Furthermore, the prices of the fossil fuels, mostly used by means of transport, have been continuously increasing and are expected to do so in the near future. Thus, it is necessary to achieve both a maximum efficiency and as a reduction of pollutant emissions in diesel engines. To accomplish this goal, the understanding of fuel injection processes and subsequent fuelair mixing formation is essential because they play a major role in combustion and also 


\begin{tabular}{|c|c|c|}
\hline \multicolumn{3}{|c|}{ NOMENCLATURE } \\
\hline$A$ & surface area production rate & $V_{s}$ coalescence interface destruction \\
\hline$a$ & surface area production rate & coefficient \\
\hline$C_{\epsilon}$ & $\begin{array}{l}\text { modeling constant for turbulence } \\
\text { closure }\end{array}$ & $Y$ liquid fraction \\
\hline$D$ & Nozzle orifice outlet diameter & Greek Symbols \\
\hline$D_{\Sigma}$ & suitable diffusion coefficient & $€$ turbulent dissipation rate \\
\hline$h$ & enthalpy & $\mu$ viscosity \\
\hline$k$ & turbulent kinetic energy & $\rho$ density \\
\hline$L$ & Nozzle orifice length & $\tau$ time scale \\
\hline$M W_{F}$ & molecular weight of fuel & $\Sigma$ mean interfacial surface-area density \\
\hline$M W_{\mathrm{amb}}$ & $\begin{array}{l}\text { molecular weight of all gaseous } \\
\text { ambient species }\end{array}$ & $\sigma \quad$ surface-tension coefficient \\
\hline$\dot{m}$ & air entrainment & Superscript \\
\hline$\dot{m}_{0}$ & mass fuel flow at the nozzle exit & ' fluctuating quantity \\
\hline$p$ & pressure & \\
\hline$p_{v}$ & vapor pressure & Subscript \\
\hline$r$ & Nozzle orifice inlet radius & $g$ gas quantity \\
\hline $\mathrm{Sc}$ & Schmidt number & $l \quad$ liquid quantity \\
\hline
\end{tabular}

pollutant formation. Therefore, an accurate prediction of these processes is required to produce reliable engine performance and emissions predictions. Diesel spray modeling is still a challenging task due to the complex interrelated phenomena involved; some of them, such as primary atomization (Gorokhovski and Herrmann, 2008) and nozzle cavitation (Schmidt and Corradini, 2001), are not fully understood.

Diesel spray modeling has been carried out by means of the discrete droplet method (DDM) (Dukowicz, 1980) for more than 30 years. This method applies a Lagrangian description of the liquid spray, using parcels (statistical representations of a number of individual fuel droplets with the same properties) that are injected in the gaseous phase. The DDM method presents some well known drawbacks for dense two-phase flow modeling. Some basic hypotheses, such as low liquid volume fraction or homogeneously distributed parcels in the computational cells, are not valid in the near nozzle flow of diesel sprays. It is often necessary to use grid sizes larger than the orifice diameter, resulting in inadequately resolved flow structures, to prevent some numerical stability problems (Abraham, 1997; Iyer and Abraham, 1997). These issues usually require a "bestpractice" approach when using this method (Abraham and Picket, 2010; Lucchini et al., 2011; Som and Aggarwal, 2009). A recent work by Senecal et al. (2012) achieved gridconvergent diesel spray DDM simulations using an improved particle-tracking algorithm 
(Are et al., 2005). This showed that grid-dependency issues usually reported can be overcome, but not other DDM approach limitations for dense spray simulations.

Experimental measurements conducted by Siebers and simple 1-D models (Siebers, 1998, 1999, 2008) show that characteristic vaporization lengths in DI diesel sprays can be predicted by the means of mixing-controlled assumptions. An implication of these finding is that under current diesel injection conditions, turbulent mixing and gas entrainment may be the dominant phenomena with respect to fuel vaporization. Taking that into account, a model that emphasizes them would be a potentially better approach. Thus the $\Sigma$-Y atomization model (Vallet and Borghi, 1999) has emerged for diesel spray simulations (Desportes et al., 2010; García-Oliver et al., 2013; Lebas et al., 2009; Ning et al., 2009; Salvador et al., 2014; Xue et al., 2014) as a promising alternative to the classical Lagrangian models. In this kind of model, an Eulerian description is applied to solve the two-phase flow together with an interface surface density equation to compute the extent of the atomization process. It is a natural approach for including nozzle geometry flow effects on spray calculations (Ning et al., 2009) and a more suitable description for the primary atomization occurring in the near-field of diesel sprays (Blokkeel et al., 2003; Xue et al., 2014).

In the present work, two different spray modeling approaches, namely a fully Eulerian and an Eulerian-Lagrangian one, are compared using the OpenFOAM (Weller et al., 1998) computational fluid dynamics (CFD) Framework against experimental measurements available from the database of the Engine Combustion Network (ECN, 2012). ECN has the purpose of coordinating international collaboration among experimental and computational researchers in the complex area of engine combustion research. The data were obtained from well-documented experiments made on specific rigs for spray characterization. These data include both global and local parameters, such as tip penetration (vapor and liquid fuel phases) and fuel vapor mixture fraction, over a wide range of ambient and injection conditions. Furthermore, measurements from the Advanced Photon Source (APS) at Argonne National Laboratory (ANL) (Kastengren et al., 2012b) have also been used, which provide unique near nozzle projected liquid mass distribution. All these data together enable a complete and detailed model validation and evaluation. The aim of the article is to use the ECN measurements as a reference of comparison between the two different CFD approaches for modeling diesel sprays and to evaluate the advantages of the $\Sigma$-Y eulerian model over the DDM method.

\section{MODEL DESCRIPTION}

\section{$2.1 \Sigma$-Y Model}

The $\Sigma$-Y model considers the liquid-gas mixture as a pseudo-fluid with a single velocity field. Under the assumption that the flow exiting the injector is operating at large Reynolds and Weber numbers, it is possible to assume a separation of the large scale 
flow features, such as mass transport, from the atomization process occurring at smaller scales. This allows the direct simulation of the large scale bulk transport of the liquid while unresolved turbulent transport is modeled using standard closures such as those used in Reynolds-averaged turbulence models.

To track the dispersion of the liquid phase, an indicator function is used taking a value of unity in the liquid phase and zero in the gas phase. The mean liquid volume fraction is denoted $(\bar{Y})$ and the mean mass averaged fraction is defined as $(\tilde{Y}=\overline{\rho Y} / \bar{\rho})$. Favre averaging the transport equation for the liquid mass fraction yields,

$$
\frac{\partial \bar{\rho} \tilde{Y}}{\partial t}+\frac{\partial \bar{\rho} \tilde{u}_{i} \tilde{Y}}{\partial x_{i}}=-\frac{\partial \bar{\rho} \widetilde{u_{i}^{\prime} Y^{\prime}}}{\partial x_{i}}-\bar{\rho} \tilde{\dot{Y}}_{\text {vap }}
$$

where $u^{\prime}$ denotes the density weighted turbulent fluctuations in velocity, $Y^{\prime}$ denotes turbulent fluctuations in liquid mass fraction, and the last term accounts for vaporization, which will be discussed later. The turbulent diffusion liquid flux captures the effect of the relative velocity between the two phases (Vallet et al., 2001). While the approach used here assumes that the resolved momentum of the liquid-gas mixture can be characterized by a single bulk velocity, the slip velocity can be expressed explicitly as derived by Demoulin et al. (2007):

$$
\left.u_{i}\right|_{l}-\left.u_{i}\right|_{g}=\frac{1}{\tilde{Y}(1-\tilde{Y})} \cdot \widetilde{u_{i}^{\prime} Y^{\prime}}
$$

Under the assumption that the two phases form an immiscible mixture, the massaveraged value of the indicator function is related to the density by

$$
\frac{1}{\bar{\rho}}=\frac{\tilde{Y}}{\rho_{l}}+\frac{1-\tilde{Y}}{\rho_{g}}
$$

An equation of state is then assigned to each phase. The gas phase obeys an ideal gas law, while the liquid phase is estimated following the Hankinson-Brobst-Thomson (HBT) correlation (Reid et al., 1987), in which the liquid density is a function of temperature and pressure.

To finally close the preceding system of equations, the temperature is obtained from a bulk mixture enthalpy equation, and closures must be given for the terms resulting from Favre averaging. The closure in the liquid mass transport equation is assigned using a standard turbulent gradient flux model,

$$
\bar{\rho} \widetilde{u_{i}^{\prime} Y^{\prime}}=\frac{\mu_{t}}{\operatorname{Sc}} \frac{\partial \tilde{Y}}{\partial x_{i}}
$$

and the closure corresponding to the Reynolds stresses in the momentum equation is given by a standard $k-\varepsilon$ model (Launder and Spalding, 1972). 
The solution of the preceding equations fully characterizes the large-scale bulk motion of the flow. Several other options exist for obtaining closure in this system of equations [see, e.g., the discussion in Demoulin et al. (2007) and Trask et al. (2012)]. Conversely, the small scale atomization is modeled by solving a transport equation for the evolution of the density of interphase surface area $\Sigma$, originally proposed by Vallet and Borghi (1999). The evolution equation is given by

$$
\frac{\partial \bar{\Sigma}}{\partial t}+\frac{\partial \tilde{u}_{j} \bar{\Sigma}}{\partial x_{j}}=\frac{\partial}{\partial x_{j}}\left(D_{\Sigma} \frac{\partial \bar{\Sigma}}{\partial x_{j}}\right)+(A+a) \bar{\Sigma}-V_{s} \bar{\Sigma}^{2}-\overline{\dot{\Sigma}}_{\text {vap }}
$$

A detailed explanation of the different terms of the previous equation can be found in García-Oliver et al. (2013).

To account for spray evaporation, both an additional transport equation for vapor fuel mass fraction and a procedure for calculating the source term from Eq. (1) have to be added to the model. The transport equation can be written in a similar way to the conservation of liquid fuel as

$$
\frac{\partial \bar{\rho} \tilde{Y}_{v}}{\partial t}+\frac{\partial \bar{\rho} \tilde{u}_{i} \tilde{Y}_{v}}{\partial x_{i}}=-\frac{\partial \bar{\rho} \widetilde{u_{i}^{\prime} Y_{v}^{\prime}}}{\partial x_{i}}+\bar{\rho} \tilde{\dot{Y}}_{\text {vap }}
$$

A standard turbulent gradient law is used for closure in this transport equation, similarly to the liquid phase [Eq. (4)]. The sink and source terms for fuel liquid-vapor transport equations are calculated in terms of a rate needed to achieve local adiabatic saturation conditions. This can be written as

$$
\dot{Y}_{v}=\frac{Y_{v, \text { sat }}-\tilde{Y}_{v}}{\tau_{\text {evap }}}
$$

where $\tilde{Y}_{v}$ is the local vapor fuel mass fraction, $Y_{v \text {,sat }}$ is the value of vapor fuel mass fraction under adiabatic saturation conditions, and $\tau_{\text {evap }}$ is a relaxation time. The latter parameter has been set equal to the computational time step, so that the local value of the vapor fuel mass fraction $\tilde{Y}_{v}$ tends to the objective value $Y_{v \text {,sat }}$ in one computational time step (characteristic time of the computation).

Finally, $Y_{v \text {,sat }}$ is calculated by means of a locally homogeneous flow (LHF) approach (Faeth, 1983). According to that, state relationships are applied to describe spray thermodynamic conditions under the assumption of local thermodynamic equilibrium. The enthalpy for the mixture is expressed in the following terms:

$$
h(T)=Y \cdot h_{f, l}(T)+Y_{v} \cdot h_{f, v}(T)+\left(1-Y-Y_{v}\right) \cdot h_{a}(T)
$$

Together with the thermodynamic liquid-vapor equilibrium assumption, the local vapor pressure $\left(p_{v}\right)$ is determined from the local temperature and thus composition can be derived (Pastor et al., 2008). The equilibrium local fuel vapor mass fraction is then related to the vapor pressure by 


$$
Y_{v, \text { sat }}=\frac{M W_{F}+Y \cdot\left(M W_{\mathrm{amb}}-M W_{F}\right)}{M W_{F}+\left(p / p_{v}-1\right) \cdot M W_{\mathrm{amb}}}
$$

where $M W_{i}$ corresponds to the molecular weights of each phase. $Y_{v, \text { sat }}$ is then fed into the vaporization term of the transport equation. Further description about the numerical implementation can be found in García-Oliver et al. (2013).

\subsection{DDM Model}

The solver dieselFoam (Kärrholm et al., 2008) from the open source CFD package OpenFOAM (Weller et al., 1998) was used for DDM spray calculations. This code solves the gas phase in a Eulerian framework, while the liquid fuel spray is treated by a standard DDM approach (Dukowicz, 1980). Parcels, representing a class of identical noninteracting spray droplets, are tracked through the physical space in a Lagrangian way, taking into account the mass, momentum, and energy exchange with the gas phase. Furthermore, several submodels are applied by the dieselFoam solver to model the physics of the fuel spray, and the adopted turbulence model accounts for the turbulent mixing of fuel and air. This turbulent flow is modeled via Reynolds averaged Navier Stokes (RANS). There are no source terms in the turbulence equations in this solver due to spray interaction, and therefore turbulence is only influenced by the Lagrangian phase through the momentum transfer and generation of velocity gradients (Kärrholm et al., 2008). The turbulence model used in this study was the standard $k$ - $\epsilon$ model.

The Kelvin-Helmholtz/Rayleigh-Taylor (KH-RT) hybrid model (Beale and Reitz, 1999) was adopted in this work. The fundamental mechanisms that govern spray breakup in the model are the Kelvin-Helmholtz and Rayleigh-Taylor instabilities. In the KelvinHelmholtz model, the stability analysis provides a dispersion equation that relates the growth of an initial perturbation of infinitesimal amplitude on a liquid surface to its wavelength and other physical and dynamic parameters of both the injected liquid and the ambient gas. The rate of change of the radius of the parent droplet is calculated using

$$
\frac{d r}{d t}=\frac{r-r_{c}}{\tau_{\mathrm{KH}}}
$$

where $r_{c}$ is the newly created parcel radius and $\tau_{\mathrm{KH}}$ is the breakup time defined by

$$
\tau_{\mathrm{KH}}=\frac{3.726 B_{1} r}{\Omega_{\mathrm{KH}} \Lambda_{\mathrm{KH}}}
$$

where $B_{1}$ is a calibration constant and $\Omega_{\mathrm{KH}}$ and $\Lambda_{\mathrm{KH}}$ are the frequency of the fastestgrowing wave and its corresponding wavelength, respectively.

The Rayleigh-Taylor model is based on a second type of instability associated with the rapid deceleration of the droplets. If the wavelength $\Lambda_{\mathrm{RT}}$ of the faster-growing wave is smaller than the droplet diameter, the RT waves start to grow on the droplet surface. The 
lifetime of the growing RT waves is tracked from then on, and when the lifetime exceeds the characteristic breakup time defined in Eq. (12), a catastrophic breakup occurs and the radius of the new droplet is given by Eq. (13)

$$
\begin{gathered}
\tau_{\mathrm{RT}}=\frac{C_{\tau}}{\Omega_{\mathrm{RT}}} \\
r_{c}=\frac{\pi C_{\mathrm{RT}}}{K_{\mathrm{RT}}}
\end{gathered}
$$

where $\Omega_{\mathrm{RT}}$ is the frequency of the fastest-growing wave, $K_{\mathrm{RT}}$ is the wave number, and $C_{\tau}$ and $C_{\mathrm{RT}}$ are model constants.

It is important to note that the concept of liquid core length was introduced in the original KH-RT hybrid model of OpenFOAM. Assuming that a liquid core exists in the near nozzle region, this means that the spray breakup occurs in a different way before and after the breakup length. With this concept, the RT model only affects the droplets beyond the breakup length. Breakup length is defined by Levich (1963) as

$$
L_{C}=\mathrm{CBU} \cdot d_{0} \sqrt{\frac{\rho_{l}}{\rho_{g}}}
$$

where $d_{0}$ and CBU are the nozzle diameter and the calibration constant, respectively. Parcels within the intact core of the liquid fuel jet are only affected by atomization but neither by drag nor by evaporation, while droplets outside this core length are subjected to breakup, drag, and evaporation. A similar modeling approach is presented in Lucchini et al. (2011).

Regarding droplet evaporation, the Ranz-Marshall model was selected (Crowe et al., 1997). Neither droplet collision nor coalescence was taken into account in this study.

\section{EXPERIMENTAL DATA}

To evaluate the models, the ECN Spray A database (ECN, 2012; Kastengren et al., 2012a) has been used. The "Spray A" condition consists of a free diesel spray injected into a quiescent environment, where well-defined boundary conditions and experimental data are available for model validation purposes. The nominal condition for Spray A corresponds to $150 \mathrm{MPa}$ injection pressure, $900 \mathrm{~K}$ ambient temperature, and 22.8 $\mathrm{kg} / \mathrm{m}^{3}$ as ambient density. Parametric variations are performed based on this reference case.

Detailed internal nozzle geometric characterization has been performed for the injectors employed in these experiments, where the main characteristics are presented in Table 1. $D, L$, and $r$ denote nozzle orifice outlet diameter, length, and inlet radius, respectively. The nozzle convergence is described by the $k$-factor, as defined in Macián et al. (2003). 
TABLE 1: Nozzle geometric characteristics for vaporizing sprays (ECN injectors)

\begin{tabular}{|ccccc|}
\hline Injector serial no. & $D(\mathbf{m m})$ & $L / D$ & $r / D$ & $k$-factor \\
\hline 210675 & 0.0894 & 11.5 & 0.23 & 2.7 \\
210677 & 0.0837 & 12.3 & 0.18 & 3.2 \\
\hline
\end{tabular}

In addition to standard spray characterization parameters such as liquid length, measured using diffused back-illumination (DBI), and vapor tip penetration, performed by means of Schlieren imaging (Bardi et al., 2012), a remarkable feature is that local airfuel ratio measurements have been performed using the Rayleigh scattering technique (Pickett et al., 2011). The latter data enable a complete analysis for validation and evaluation of CFD models, both in global and local terms.

Additionally, the Spray A condition of ECN is also used to evaluate the models in terms of near-field structure (dense region) of diesel sprays. This experiment is conducted with the ambient gas at room temperature $(303 \mathrm{~K})$ due to the X-ray transparent polymer windows used, which cannot be used at high temperature. Nevertheless, the same ambient density of the nominal evaporating Spray A condition is matched to reproduce similar conditions for the spray breakup process, assuming that density is a more critical parameter than pressure for atomization (Naber and Siebers, 1996). X-ray radiography measurements available in the ECN Spray A database for this condition provide unique insights into the internal structure of diesel sprays and are a most valuable tool for CFD model developers.

\section{MODEL SETUP}

A series of studies have been run with both CFD models. The injector serial and the ambient conditions of every case are presented in Table 2.

TABLE 2: Simulated cases conditions (ECN injectors)

\begin{tabular}{|c|c|c|c|}
\hline Injector serial no. & $P_{\text {inj }}(\mathbf{M P a})$ & $T_{\mathrm{amb}}(\mathbf{K})$ & $\rho_{\mathrm{amb}}\left(\mathbf{k g} / \mathbf{m}^{3}\right)$ \\
\hline \multirow[t]{2}{*}{210677 (Baseline) } & 150 & 900 & 22.8 \\
\hline & Near-field & Study & \\
\hline \multirow[t]{2}{*}{210675} & 150 & 303 & 22.8 \\
\hline & Ambient conditions & Study & \\
\hline \multirow[t]{2}{*}{210677} & 150 & 1100 & 15.2 \\
\hline & Injection pressure & Study & \\
\hline 210675 & 150 & 900 & 22.8 \\
\hline 210675 & 100 & 900 & 22.8 \\
\hline 210675 & 50 & 900 & 22.8 \\
\hline
\end{tabular}




\section{1 $\Sigma$-Y Model}

To simulate the diesel sprays with the $\Sigma$-Y model, a 2-D axisymmetric computational domain is used, considering the nozzle geometry and RANS turbulence modeling approach. The mesh is structured with nonuniform grid resolution. There are 10 cells along the orifice diameter, keeping an aspect ratio close to 1 in the near nozzle region, as depicted in Fig. 1. The nonuniform grid resolution consists of cells with an expansion ratio of 1.01 and 1.06 in the axial and radial directions, respectively. This size distribution was chosen, and used in the following calculations, after a mesh size convergence study performed due to the capability of the model to allow mesh refinement to achieve grid independent results.

The discretization of the divergence terms was solved with a Gamma NVD scheme, and a first-order Euler scheme is applied for time derivative terms. The inlet velocity boundary condition is obtained from mass flow rate and momentum flux measurements, applying a constant radial profile of axial velocity and density at nozzle outlet. The turbulent intensity was set to $5 \%$ and the length scale to $10 \%$ of nozzle diameter. Owing to the well-known round jet spreading overprediction of $k$ - $\epsilon$ type models (Pope, 1978), a corrected value (1.60) for $C_{1 \epsilon}$ is used. Pope (1978) has previously suggested that the latter value should be used for round jets. The liquid turbulent flux closure (Lebas et al., 2009 ) is calculated by means of a gradient closure with $\mathrm{Sc}_{t}=0.9$.

With this setup spray tip penetration, fuel mass fraction field, and quasi-steady liquid length can be accurately predicted for a large range of ambient gas conditions that are normally present in diesel engines. More details on the setup with the $\Sigma$-Y model can be found in García-Oliver et al. (2013).

\subsection{DDM Model}

In this work, the model domain used to perform all the DDM calculations, as in the case of the other CFD approach, consists of a 2-D axisymmetric structured mesh. Based on a

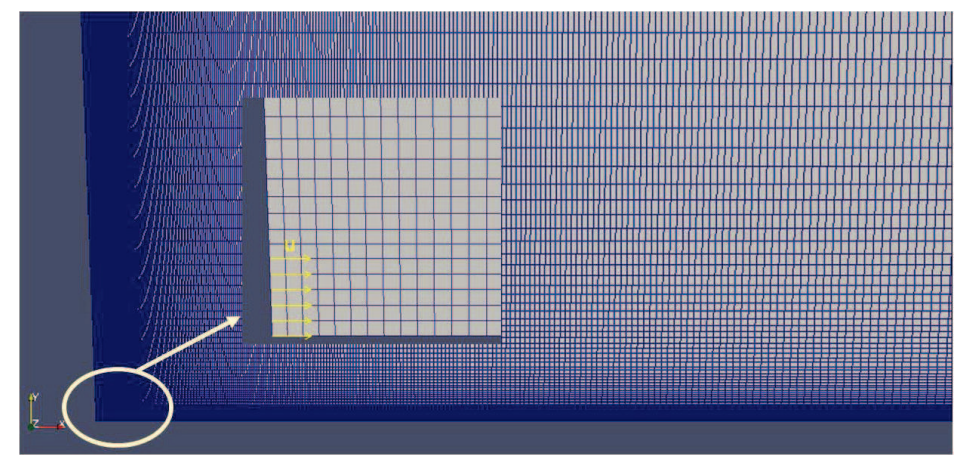

FIG. 1: Computational grid for $\Sigma$-Y model simulations. The inset shows the mesh near the nozzle exit. 
model setup proposed by López et al. (2013), a calibration process, including mesh size, turbulence model, and spray submodels constants, was carried out to determine the best parameters to properly reproduce the reference case of the Spray A condition.

Because of the high sensitivity of the Lagrangian submodels to the cell size in connection with the time step size, the cell size was determined in a parametric validation process. In this calibration study, four different meshes were evaluated to model the Spray A base condition, a coarse grid with square $0.5 \mathrm{~mm}$ cells, a refined grid in radial direction with $0.5 \times 0.25 \mathrm{~mm}$ cells, a refined grid in both directions with $0.25 \mathrm{~mm}$ squared cells, and a more refined grid in radial direction with $0.25 \times 0.125 \mathrm{~mm}$ cells. These grid resolutions are typical for RANS spray simulations as described in Lucchini et al. (2011) and Senecal et al. (2012).

In Fig. 2(left), vapor and liquid penetration predicted by the DDM model using the four different meshes are compared with the experimental measurements. In addition to this, an analysis of the air entrainment process is made in terms of the total to initial mass flux ratio $\left(\dot{m} / \dot{m}_{0}\right)$ to better understand the model predictions in Fig. 2(right). This air entrainment is computed as a function of axial distance, considering that the spray radial limit is located at the radial position where the velocity is equal to $1 \%$ of the on-axis velocity.

Penetration results show that the coarse mesh is not good enough for this simulation and both vapor and liquid penetration are underpredicted. Entrainment plots show that the coarse mesh results in a significantly higher entrainment in the near-nozzle region, but further downstream the entrainment rate (i.e., a slope in the curve) is lower than for the other three cases. This can be explained as a result of faster momentum transfer in the near-nozzle region from the fuel to the air in the coarse grid, and because of that, a
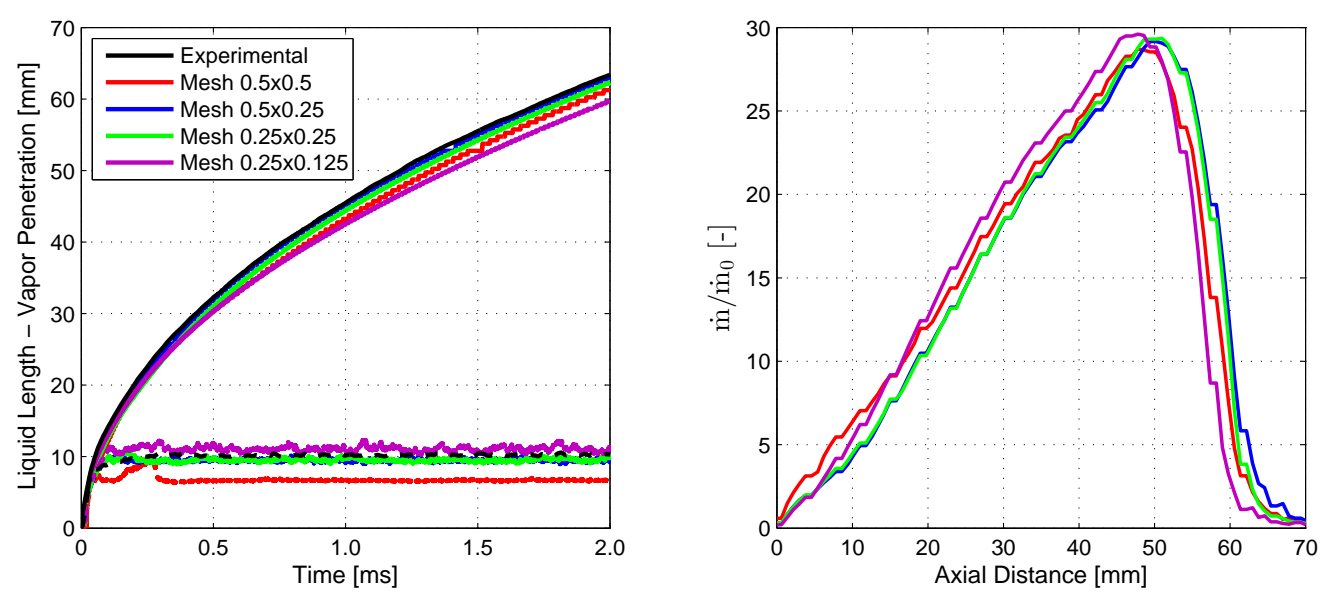

FIG. 2: (left) Vapor penetration and liquid length for DDM solver simulations and (right) computed air entrainment normalized at $2.0 \mathrm{~ms}$ after SOI, mesh calibration: Injector 210677. $P_{\text {inj }}=150 \mathrm{MPa}, T_{a}=900 \mathrm{~K}$, and $\rho_{a}=22.8 \mathrm{~kg} / \mathrm{m}^{3}$. 
lower penetration is reached compared to the other three cases. On the other hand, when the most refined mesh is used, a slightly lower axial air entrainment in the near nozzle region of the jet is reached, therefore liquid droplets are transported farther from the injector nozzle before their complete evaporation and thus the predicted liquid penetration is scarcely higher. However, once the droplets have been evaporated, the air entrainment rate increases to higher values than for the other cases, even than the coarse mesh one, and therefore penetration tends to be underpredicted. In this case, it has to be noted that with higher spatial resolution, the volume fraction of the computational cell occupied by the liquid becomes too large and, as a result, the main hypotheses of the Lagrangian approach are not fulfilled. This could explain the noticed lack of convergence in the results. Similar trends with a highly refined grid resolution were observed in Lucchini et al. (2011). The two intermediate meshes produce very similar results. Nevertheless, the vapor penetration predicted using the mesh with the grid resolution of $0.5 \mathrm{~mm}$ in axial direction and $0.25 \mathrm{~mm}$ in radial direction is slightly better and, also, the computational cost is lower. Thus, this mesh was chosen for the simulations with the DDM CFD model. It is also worthy of mention that Senecal et al. (2012) also proposed $0.25 \mathrm{~mm}$ grid size for Spray A simulations after their grid-convergency study previously mentioned. It is important to highlight the large differences in mesh resolution obtained with both CFD approaches, with a minimum grid size of $8.4 \mu \mathrm{m}$ for the $\Sigma$-Y model and $250 \mu \mathrm{m}$ for the DDM model, which unequivocally should have some influence on the adequate representation of diesel sprays of each approach.

Note, also that the number of injected parcels was set to maintain a value of $6 \times$ $10^{7}$ parcels per second. Finally, the discretization of the divergence terms was solved with a second-order scheme, and a first-order Euler scheme is applied for time derivative terms.

As previously explained, the standard $k-\varepsilon$ model was used for turbulent gaseous flow modeling, but the model parameter $C_{1 \epsilon}$ has to be set to 1.52, as suggested in López et al. (2013), to achieve a better agreement between modeling and experimental results concerning liquid and spray penetration. In Fig. 3(right), the mass flux ratio results are shown, together with vapor penetration Fig. 3(left), indicating that the spray penetration is overpredicted if the same value than in the $\Sigma$-Y model simulation is used, due to a too low mass entrainment. The results for the $C_{1 \epsilon}=1.52$ are quite accurate.

The effect of atomization and breakup submodels was also evaluated. First, the breakup length model parameter (CBU) was investigated (Fig. 4). Three conditions are evaluated: no breakup core length $(\mathrm{CBU}=0)$, a liquid core length equal to 3 times the equivalent diameter $(\mathrm{CBU}=3)$ and a value of 7 times the equivalent diameter $(\mathrm{CBU}=7)$. The validation showed that simulation results best approximate the experimental vapor penetration for the parameter equal to 3 , but the liquid length prediction is slightly better for the case of $\mathrm{CBU}=7$. In the end, the selected value for the breakup length model parameter was 3 because with this value, the liquid core length is in the order of $2 \mathrm{~mm}$, which is in the range estimated by recent analyses in Pickett et al. (2014). 

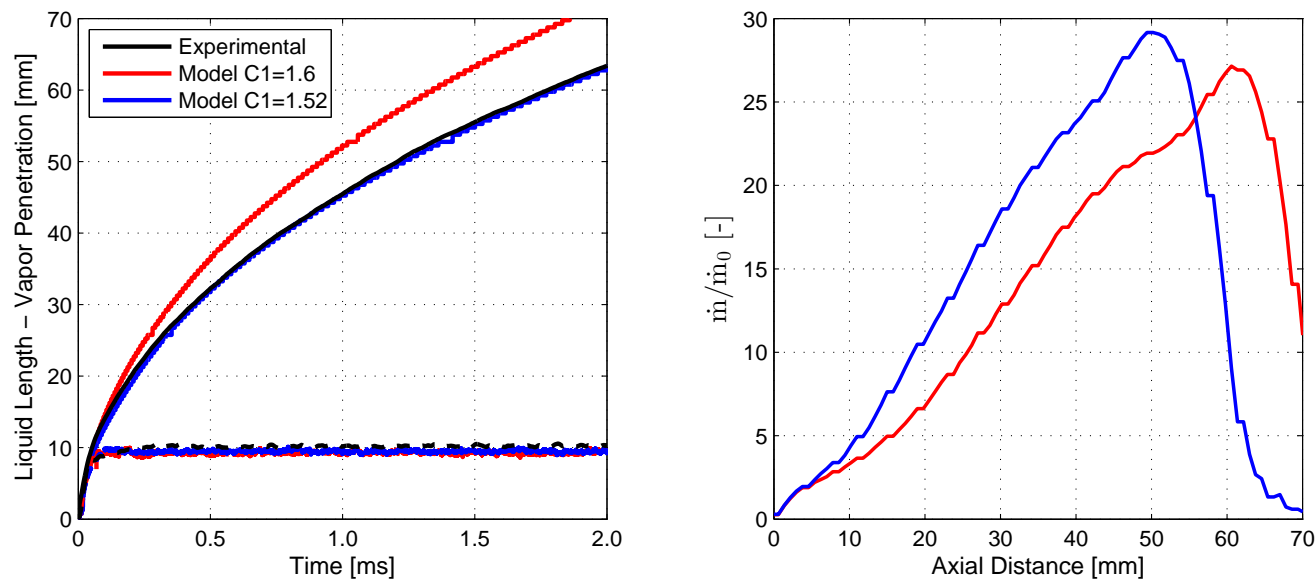

FIG. 3: (left) Vapor penetration and liquid length for DDM solver simulations and (right) computed air entrainment normalized at $2.0 \mathrm{~ms}$ after SOI, turbulence calibration: Injector 210677. $P_{\text {inj }}=150 \mathrm{MPa}, T_{a}=900 \mathrm{~K}$ and $\rho_{a}=22.8 \mathrm{~kg} / \mathrm{m}^{3}$.
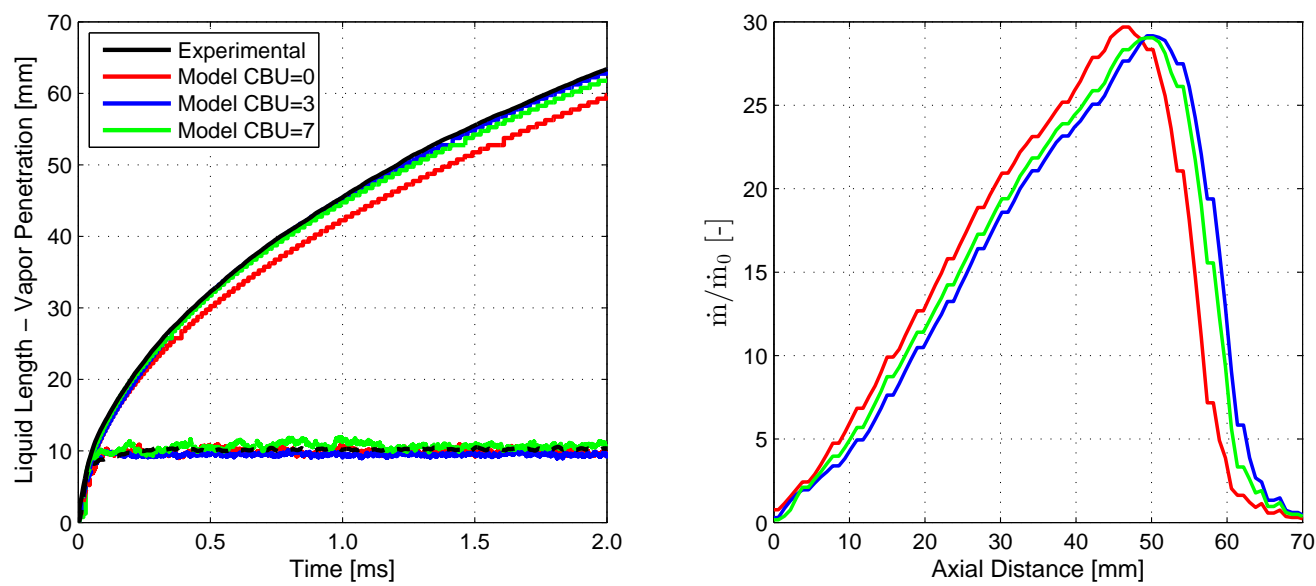

FIG. 4: (left) Vapor penetration and Liquid Length for DDM solver simulations and (right) computed Air Entrainment normalized at $2.0 \mathrm{~ms}$ after SOI, CBU constant calibration: Injector 210677. $P_{\text {inj }}=150 \mathrm{MPa}, T_{a}=900 \mathrm{~K}$, and $\rho_{a}=22.8 \mathrm{~kg} / \mathrm{m}^{3}$.

Finally, the Kelvin-Helmholtz $(\mathrm{KH})$ model constant was analyzed. Figure 5(left) shows comparisons of vapor and liquid penetration among the original B1 $=40$ and its half and twice $(\mathrm{B} 1=20, \mathrm{~B} 1=80)$ as well as experimental measurements. The vapor penetration does not show much variation with the change of B1. However, a smaller value of $\mathrm{B} 1$ results in earlier breakup, and hence the liquid penetration becomes shorter. Thus, $\mathrm{B} 1=40$ and $\mathrm{B} 1=80$ could be good options, but the original value was chosen to run all the following DDM CFD simulations. 

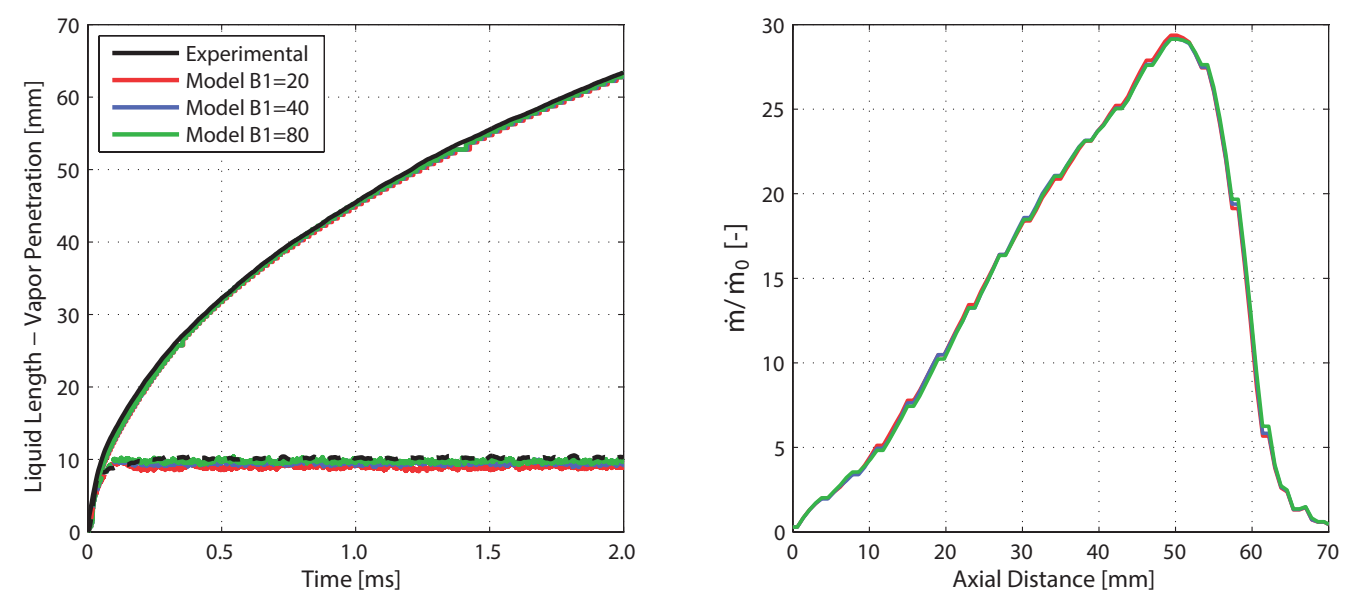

FIG. 5: (left) Vapor penetration and liquid length for DDM solver simulations and (right) computed air entrainment normalized at $2.0 \mathrm{~ms}$ after SOI, B1 constant calibration: Injector 210677. $P_{\text {inj }}=150 \mathrm{MPa}, T_{a}=900 \mathrm{~K}$, and $\rho_{a}=22.8 \mathrm{~kg} / \mathrm{m}^{3}$.

As already analyzed, in Figs. 3, 4, and 5(right), the results of axial air entrainment normalized by the mass flow at the nozzle exit are shown. Modifying the $C_{1 \epsilon}$ only affects the slope of the entrainment ratio, that is, the entrainment constant. However, modifying the breakup length model parameter (CBU), this slope is almost constant. In this case, the primary atomization process and the exchange of momentum are greatly modified. Note that different liquid core lengths are achieved with each value of the parameter, but once the flow reaches the maximum liquid length, entrainment occurs in exactly the same way; that is, the entrainment ratio curves in Fig. 4 are simply shifted in space toward locations farther from the nozzle. In contrast with this, the variation of the KelvinHelmholtz model constant (B1) produces no changes in the air entrainment profiles. The fact that an injector nozzle with a small diameter and a high injection pressure condition is used may be the explanation for this behavior. According to that, extremely fast fuel spray evaporation and momentum transfer take place, which lead to less influence on the variation of an atomization parameter.

\section{RESULTS AND DISCUSSION}

\subsection{Reference Case}

Spray A specifications were selected as the base case simulation to evaluate model predictions. Results shown in Fig. 6(left) depict good agreement for both models in terms of liquid and vapor penetration. In both cases, predictions seem to fall within the experimental uncertainty of measured values. Nevertheless, only the $\Sigma$-Y model is capable of predicting the initial evolution of the penetration [Fig. 6(right)]. This can be justified because of the fact that the Eulerian model uses a much finer grid to improve spatial 

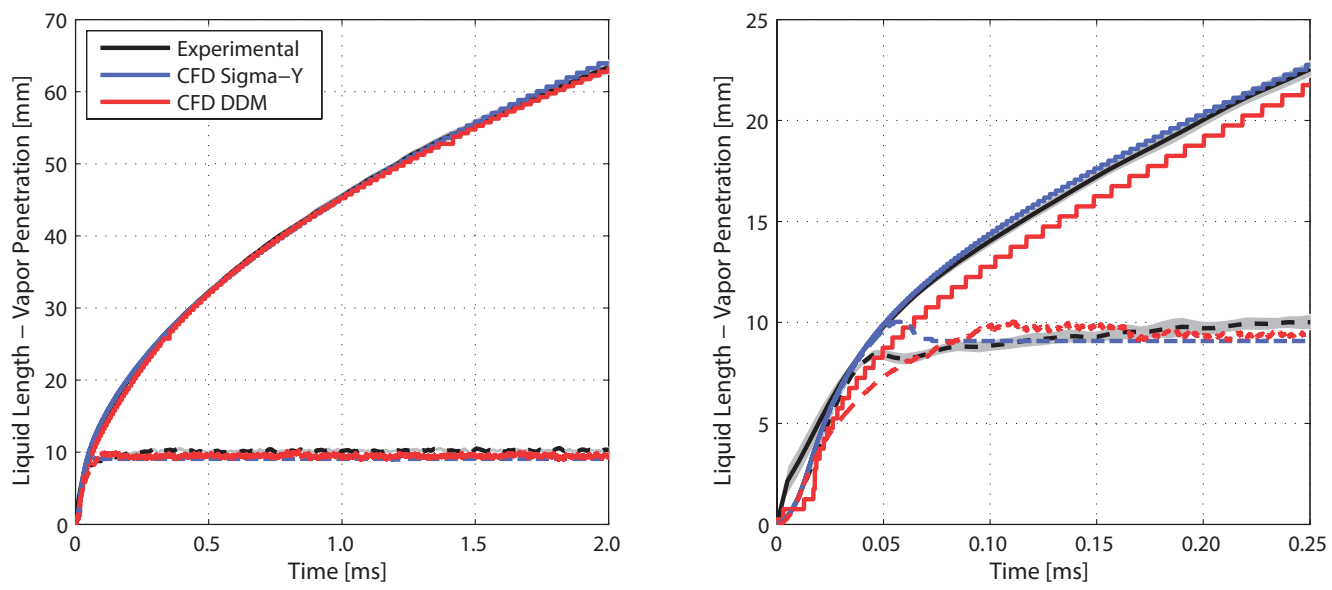

FIG. 6: Computed and measured liquid and vapor penetration: Injector $210677, P_{\text {inj }}=$ $150 \mathrm{MPa}, T_{a}=900 \mathrm{~K}$, and $\rho_{a}=22.8 \mathrm{~kg} / \mathrm{m}^{3}$. (right) Detail of the first $0.25 \mathrm{~ms}$.

resolution, which is not possible with the DDM model. In terms of liquid length, both models achieve reasonable predictions.

Predicted versus measured values of mixture fraction are shown in Fig. 7. Predicted values on the axis always fall within the confidence interval in the case of the $\Sigma$-Y model. However, the results obtained with the DDM solver have a different trend. While up to $22 \mathrm{~mm}$ and the predicted values are higher than both measured as well as $\Sigma$-Y ones, then DDM results become equal to both experimental and $\Sigma$-Y ones to finally end up with lower values, almost out of the confidence interval.

Regarding radial dispersion of mixture fraction, the shape of the profiles is adequately predicted by both models, as shown in Fig. 7(right). In this case, results have been plotted in normalized coordinates (i.e., the $y$-axis is the local mixture fraction divided by the on-axis one, while the $x$-axis is the radial divided by the axial coordinate). There is a slight bias toward narrower radial profiles in the calculations compared to the experimental ones, which should indicate less radial dispersion and hence slightly lower entrainment. At $25 \mathrm{~mm}$, both models essentially produce the same results, in agreement with the centerline evolution, but it is not the same at $45 \mathrm{~mm}$. Although CFD profiles are wider compared to the experimental ones at $25 \mathrm{~mm}$ for both CFD models, the $\Sigma-\mathrm{Y}$ predictions are slightly narrower, that is, less radial dispersion, which may be the explanation of the higher values shown on the centerline profile. Considering these results, the overall agreement of both models is quite remarkable.

Finally, for the baseline case, an analysis of axial air entrainment together with the measured spray vapor contours from Rayleigh measurements is made, as shown in Fig. 8, where solid lines correspond to contours of $1 \%$ the on-axis mixture fraction value, while dashed lines show the stoichiometric isosurface. It must be noted that Rayleigh data are only available in an interval between $20 \mathrm{~mm}$ and $50 \mathrm{~mm}$. 

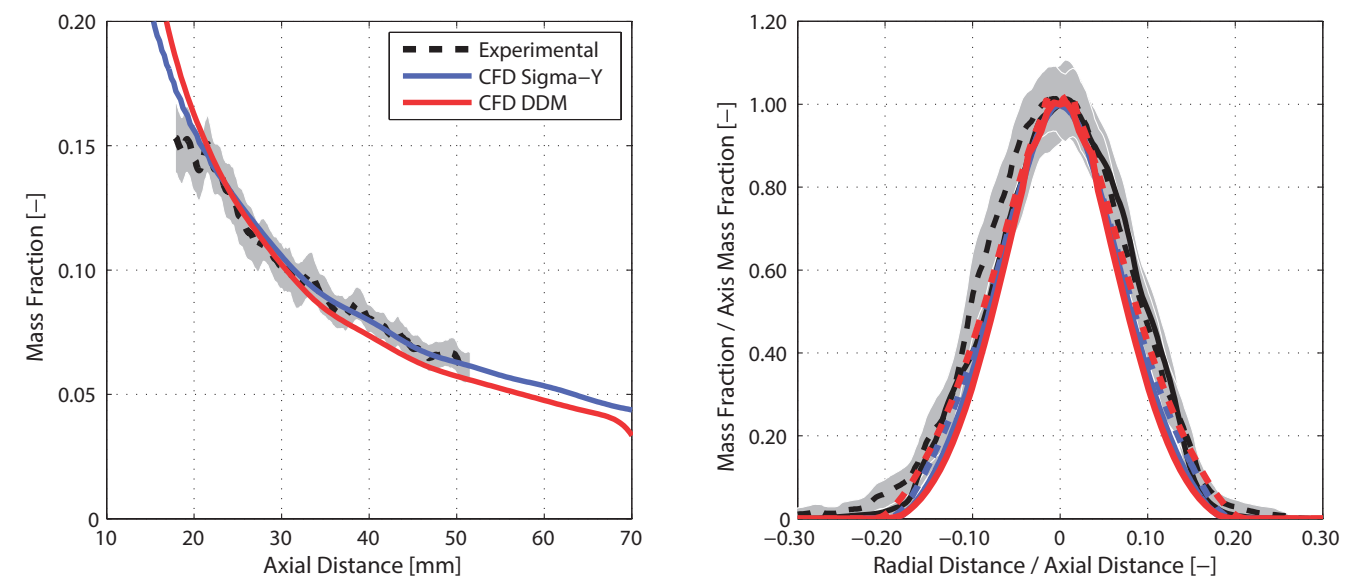

FIG. 7: (left) Computed and measured centerline mixture fraction and (right) mixture fraction radial profiles normalized at $25 \mathrm{~mm}$ (solid line) and $45 \mathrm{~mm}$ (dashed line): Injector $210677, P_{\text {inj }}=150 \mathrm{MPa}, T_{a}=900 \mathrm{~K}$, and $\rho_{a}=22.8 \mathrm{~kg} / \mathrm{m}^{3}$.
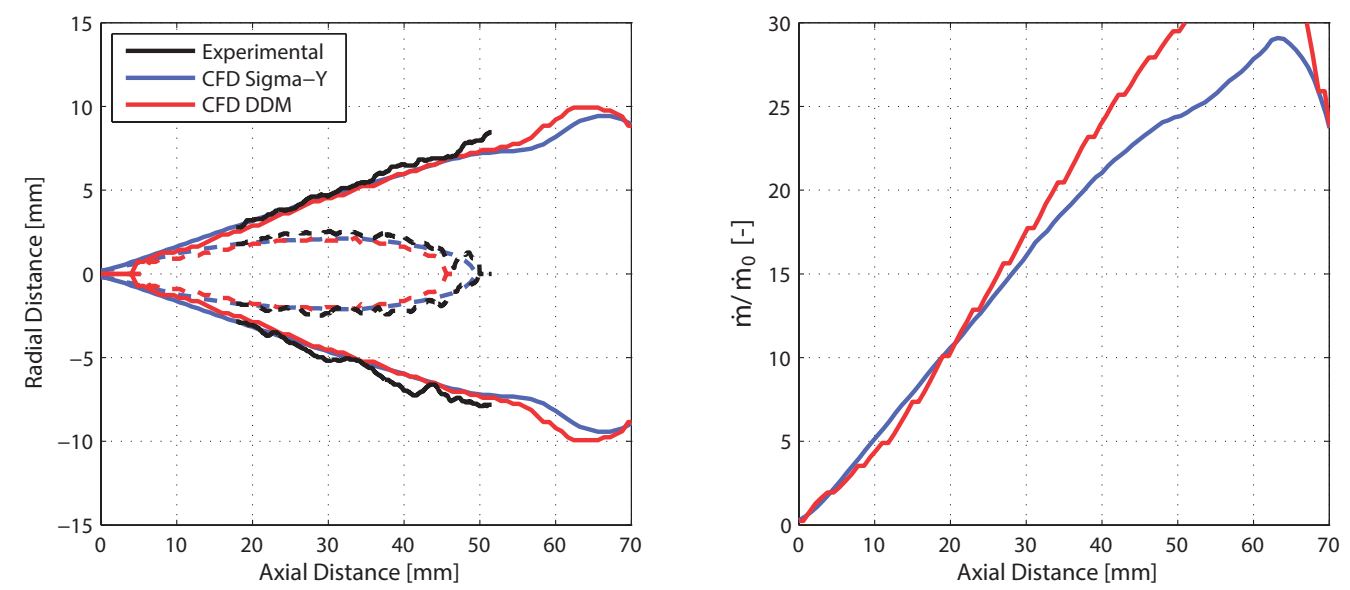

FIG. 8: (left) Computed and measured contours of vapor region and (right) computed air entrainment normalized at $3.0 \mathrm{~ms}$ after SOI: Injector $210677, P_{\mathrm{inj}}=150 \mathrm{MPa}, T_{a}=$ $900 \mathrm{~K}$, and $\rho_{a}=22.8 \mathrm{~kg} / \mathrm{m}^{3}$.

Initially, up to $30 \mathrm{~mm}$, the $\Sigma$-Y contour is slightly wider than the DDM one. At 30-50 mm, both models predict essentially the same radius, while DDM radial dispersion is higher at the nozzle tip. This radial evolution is essentially coherent with the mass flow one. The different value of the turbulence constant $C_{1 \epsilon}$ needed by the DDM model results in a more intense mixing rate that compensates the lower entrainment in the liquid-vapor zone. Despite these differences, the spray radius predicted by both models is remarkably close to the experimental data. On the other hand, noticeable 
differences appear when both models are compared with experimental data in terms of the stoichiometric isosurface. In this case, the predicted contours are almost the same up to $35 \mathrm{~mm}$, even though they are slightly narrower compared to the experimental measurements. But it is in the spatial position between $35 \mathrm{~mm}$ and $50 \mathrm{~mm}$, where the differences can be observed. Predictions by the $\Sigma$-Y model become closer to the experimental ones, while DDM predictions show a narrower stoichiometric surface due to the higher mixing rate.

\subsection{Near-Field Study}

To evaluate the capability of the models to reproduce the near-field internal structure of diesel sprays and, therefore, the atomization process, the experimental data used for comparison were obtained from the X-ray radiography measurements conducted at Argonne National Laboratory. In particular, the variable used for comparison is the projected mass density of the fuel, which is calculated by a line-of-sight integration along the X-ray beam (Kastengren et al., 2012b; Pickett et al., 2014). As a result, a similar procedure is replicated with the data from simulations to enable fair comparisons against experiments.

The simulations have been run using the same 2-D axisymmetric domain and grid resolution, inlet boundary condition, and calibrated model constants of the reference case. The purpose of this study is not to achieve the best possible results with each model but to make a comparison of the results provided by the models in terms of nearfield structure with the more suitable calibration for far-field conditions. In Fig. 9, the projected mass density along the transverse direction comparing both CFD simulation predictions and $\mathrm{X}$-ray radiography data is shown at $0.1,0.6,2.0$, and $4.0 \mathrm{~mm}$ downstream of the nozzle exit.

First of all, note that the experimental transverse distributions have been centered to compare them with the CFD axisymmetric results. At $0.1 \mathrm{~mm}$, both models predict almost the same peak projected density, but noticeable differences in width could be detected with a narrower DDM profile. At $0.6 \mathrm{~mm}$ downstream of the nozzle exit, although DDM profile width is quite similar to experiments, the peak value is underpredicted. However, predictions by the $\Sigma$-Y model are really close to the experimental values, showing a better spatial behavior reproducing the near nozzle flow structure. The largest differences can be observed at $2.0 \mathrm{~mm}$. Fuel mass projected density predictions by DDM model are largely underpredicted in terms of peak value, while $\Sigma$-Y model ones reproduce very well the experimental trend both in peak value and radial dispersion. Similar trends are observed in Xue et al. (2014), even using the same grid size for both models. Finally, at $4.0 \mathrm{~mm}$ downstream location, both models predict reasonable values of peak projected density, but it has to be noted that $\Sigma$-Y model reproduces the decrease from 2.0 to $4.0 \mathrm{~mm}$, while the DDM model keeps the value almost constant and predicts more radial dispersion. Overall, the $\Sigma$-Y model provides the best match with the experimental 

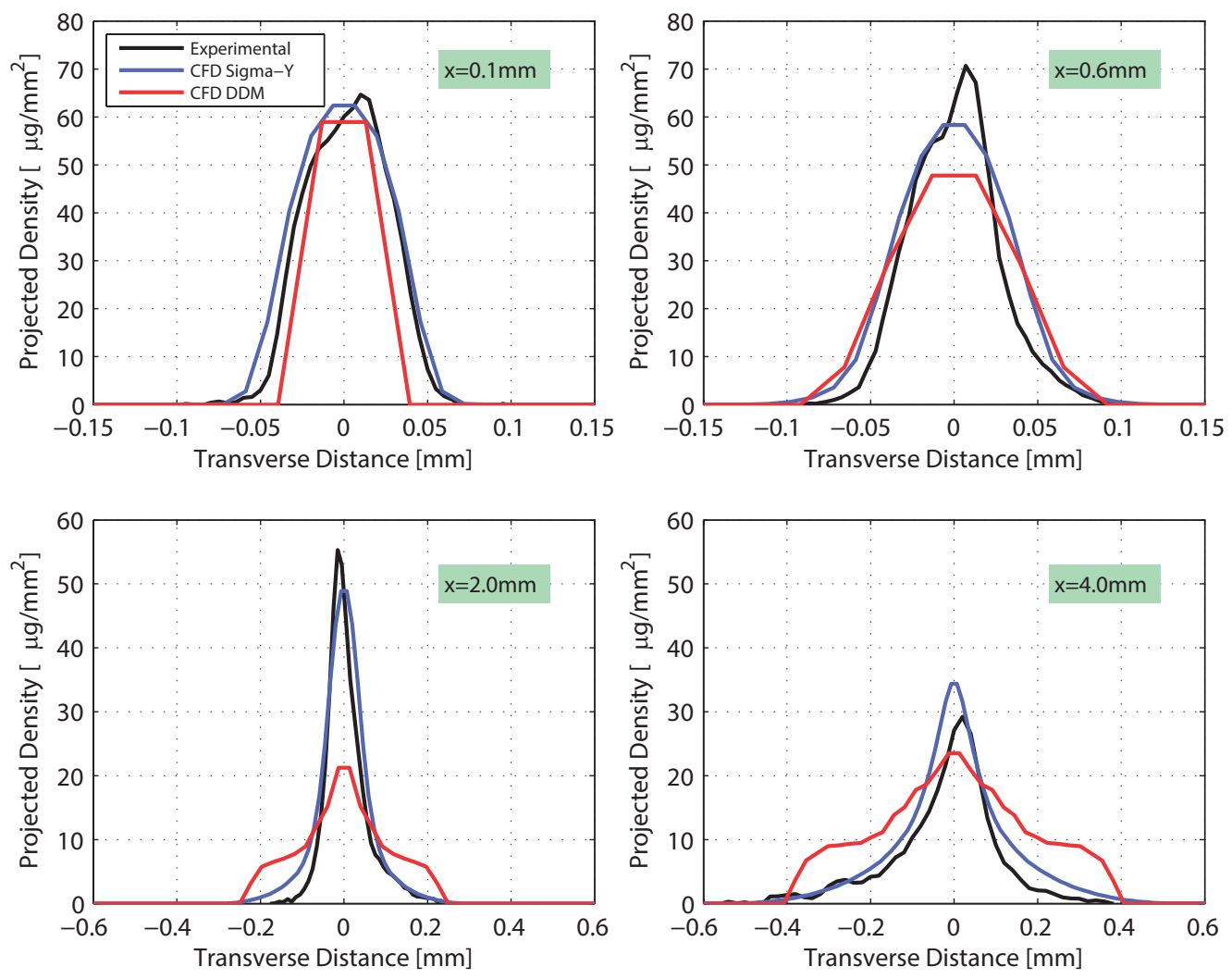

FIG. 9: Computed and measured profiles of projected mass density at $0.5 \mathrm{~ms}$ after SOI: Injector $210675, P_{\text {inj }}=150 \mathrm{MPa}, T_{a}=303 \mathrm{~K}$, and $\rho_{a}=22.8 \mathrm{~kg} / \mathrm{m}^{3}$.

data and can capture the trend of the internal structure of a diesel spray in the near-field fairly well.

\subsection{Ambient Conditions Variation}

Apart from the nominal Spray A condition, an additional operating condition $\left(P_{\text {inj }}=\right.$ $150 \mathrm{MPa}, T_{a}=1100 \mathrm{~K}$ and $\rho_{a}=15.2 \mathrm{~kg} / \mathrm{m}^{3}$ ) was selected to evaluate the performance of both models under ambient temperature and density variation. As in the baseline condition, a complete range of experimental data is available.

Results shown in Fig. 10 depict good agreement for both models in terms of vapor penetration. In both cases, the predictions are within the experimental uncertainty of measured values in an overall view. However, the predicted liquid penetration in the case of the $\Sigma$-Y model is slightly underestimated, while with the DDM model, it is overpredicted. Once again, in the early evolution of the vapor penetration in Fig. 10(right), the $\Sigma$-Y model achieves the best agreement with the experimental measurements. 

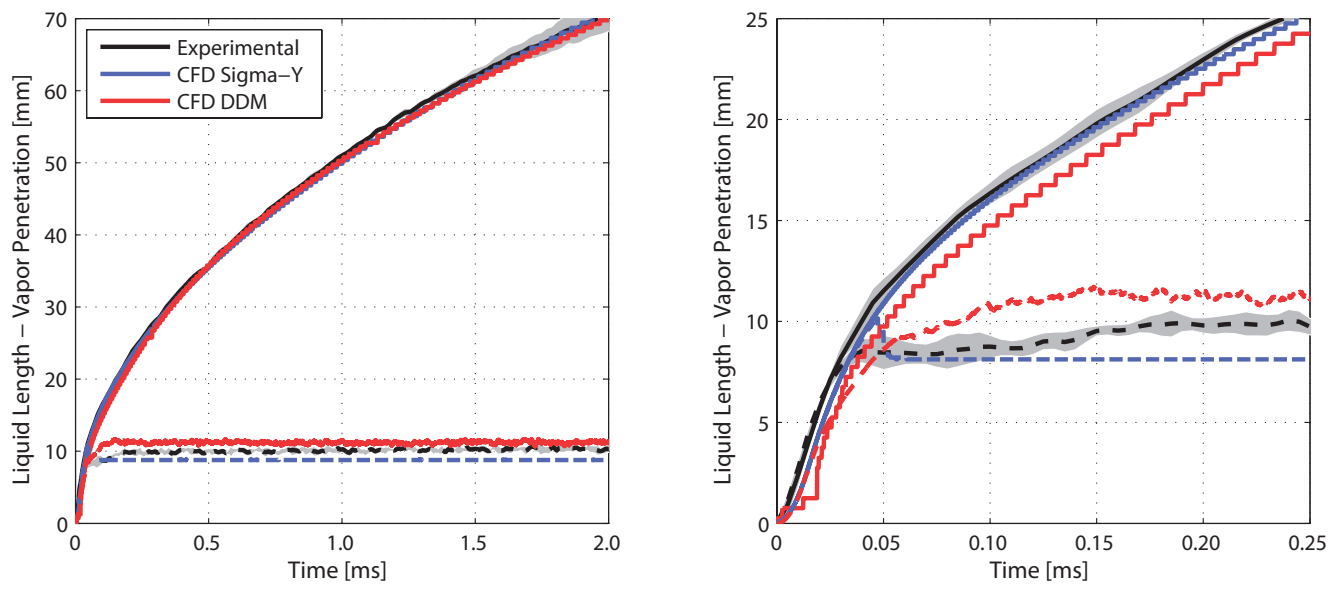

FIG. 10: Computed and measured liquid and vapor penetration: Injector $210677, P_{\text {inj }}=$ $150 \mathrm{MPa}, T_{a}=1100 \mathrm{~K}$, and $\rho_{a}=15.2 \mathrm{~kg} / \mathrm{m}^{3}$. (Right) Detail of the first $0.25 \mathrm{~ms}$.

Further experimental data are the mixture fraction profiles on the axis and the radial distribution. Predicted versus measured values of mixture fraction are shown in Fig. 11. Predicted values on the axis, Fig. 11(left), fall within the confidence interval, in the case of the $\Sigma$-Y model, up to $45 \mathrm{~mm}$. However, the results obtained with the DDM solver have a different trend. While up to almost $30 \mathrm{~mm}$ the predicted values are higher than both the experimental measurements and the $\Sigma$-Y values, the predicted axial mixture fraction profile crosses the $\Sigma$-Y one to finally end up with lower values, within the confidence interval.
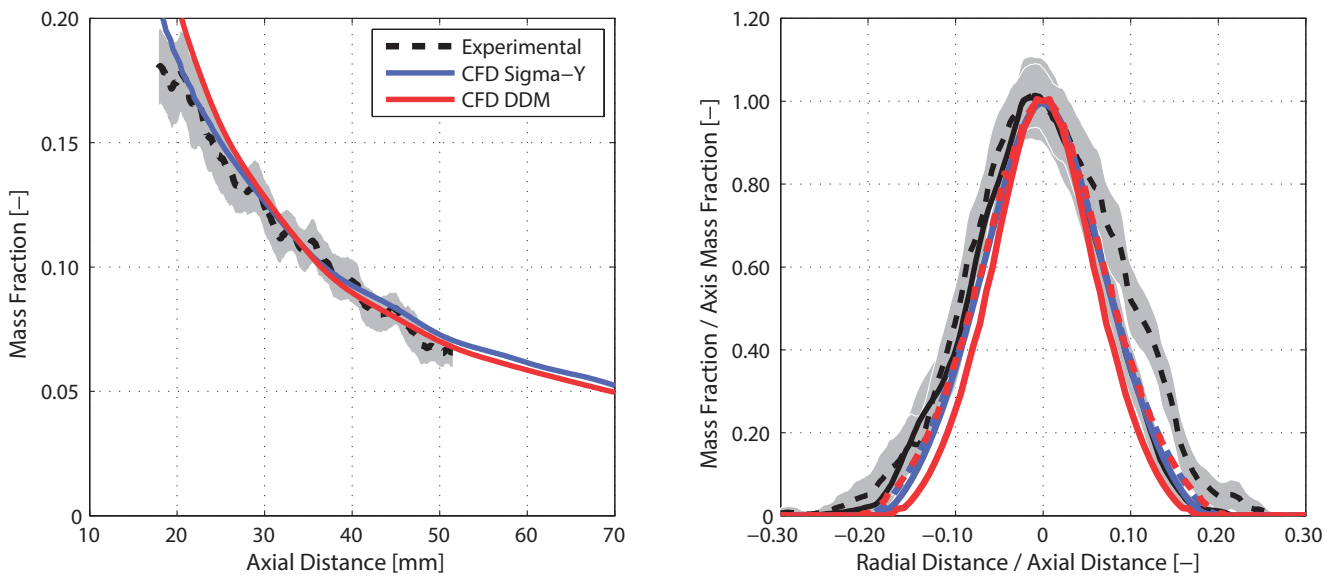

FIG. 11: (left) Computed and measured centerline mixture fraction and (right) mixture fraction radial profiles normalized at $25 \mathrm{~mm}$ (solid line) and at $45 \mathrm{~mm}$ (dashed line): Injector $210677, P_{\text {inj }}=150 \mathrm{MPa}, T_{a}=1100 \mathrm{~K}$, and $\rho_{a}=15.2 \mathrm{~kg} / \mathrm{m}^{3}$. 
Regarding radial distribution of mixture fraction, the shape of the profiles is adequately predicted by both models, as shown in Fig. 11(right). The results in this particular operating condition are very similar to the baseline case of Spray A considered in the Section 5.1. Here the main differences appear at $25 \mathrm{~mm}$, with the DDM profile clearly narrower, which explains the higher values shown on the centerline evolution. On the other hand, predicted values at $45 \mathrm{~mm}$ by both models are very similar, as could be noticed from the centerline data.

To end up with the analysis of this particular operation point, as well as in the reference case, axial air entrainment together with the measured spray vapor contours are studied, as shown in Fig. 12. From the spray origin up to $30 \mathrm{~mm}$, the modeled radial contour with the $\Sigma$-Y model is slightly wider in comparison with the predicted using the DDM solver. From 30 to $50 \mathrm{~mm}$, both approaches predict essentially the same radius. Further downstream, DDM predictions become wider, although such locations correspond to the unsteady spray head. Even though there are some differences between both CFD models, the predicted radial contours have a remarkable accuracy similarity to experimental data. In the same way as for the already analyzed baseline case, this behavior is coherent with the axial air entrainment profiles, in which mass flow is higher according with the wider spray prediction, as a result of a greater area for air entrainment. The different value of the turbulence constant $C_{1 \epsilon}$ for each case may be the explanation for this phenomenon.

Taking into account the experimental data of the vapor stoichiometric isosurface, in Fig. 12(left), some differences between both models were detected. While the radial width of the stoichiometric surface is reproduced adequately by both models over most of the length, differences appear at the furthest axial location, which cannot be validated
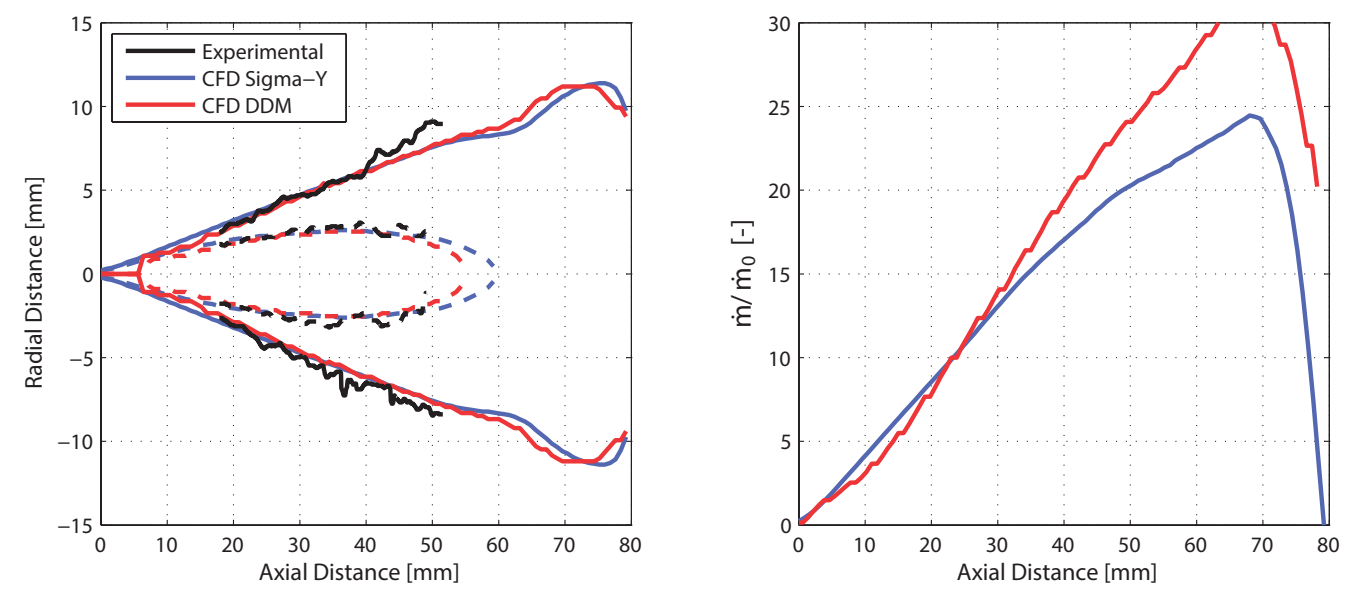

FIG. 12: (left) Computed and measured contours of vapor region and (right) computed air entrainment normalized at $3.0 \mathrm{~ms}$ after SOI: Injector $210677, P_{\mathrm{inj}}=150 \mathrm{MPa}, T_{a}=$ $1100 \mathrm{~K}$, and $\rho_{a}=15.2 \mathrm{~kg} / \mathrm{m}^{3}$. 
due to the absence of experimental data for $x \geq 50 \mathrm{~mm}$. Nevertheless, the behavior of both CFD models is the same as in the baseline condition, with $\Sigma$-Y model results slightly longer than the DDM model ones.

\subsection{Injection Pressure Variations}

Finally, parametric studies with a set of different injection conditions were performed. The results of these studies, including spray vapor penetration and liquid length predictions, have been summarized in Fig. 13. In addition to this, a detailed analysis of air entrainment has been conducted to explain some results (Fig. 14).

Experimental results show that penetration of the liquid phase does not change, however, injection pressure modifies the vapor penetration due to the modification in nozzle momentum flux. This lack of dependence could be explained because vaporization is controlled by air entrainment into the spray. For a fully developed spray, there is a linear dependence between the rate of air entrainment and both the axial velocity and the mixture fraction distributions (Siebers, 1998). As a result, for the different injection pressures, the mixing rate is the same. In addition, studies show almost no effect of injection pressure on the spray spreading angle once the spray has reached steady state.

In terms of modeling results, both CFD codes are capable of making an accurate prediction of vapor penetration in any condition. However, this is not the same for the quasi-steady values of liquid length. Predictions of the DDM solver are sensitive to variations regarding the injection pressure, instead of keeping it almost invariable as the $\Sigma-Y$ model, which depicts a good agreement with experimental measurements (Fig. 13). In Fig. 14, an analysis of air entrainment is made to better understand the penetration results and the behavior of the sprays in the two CFD models. In this figure, the mass flux
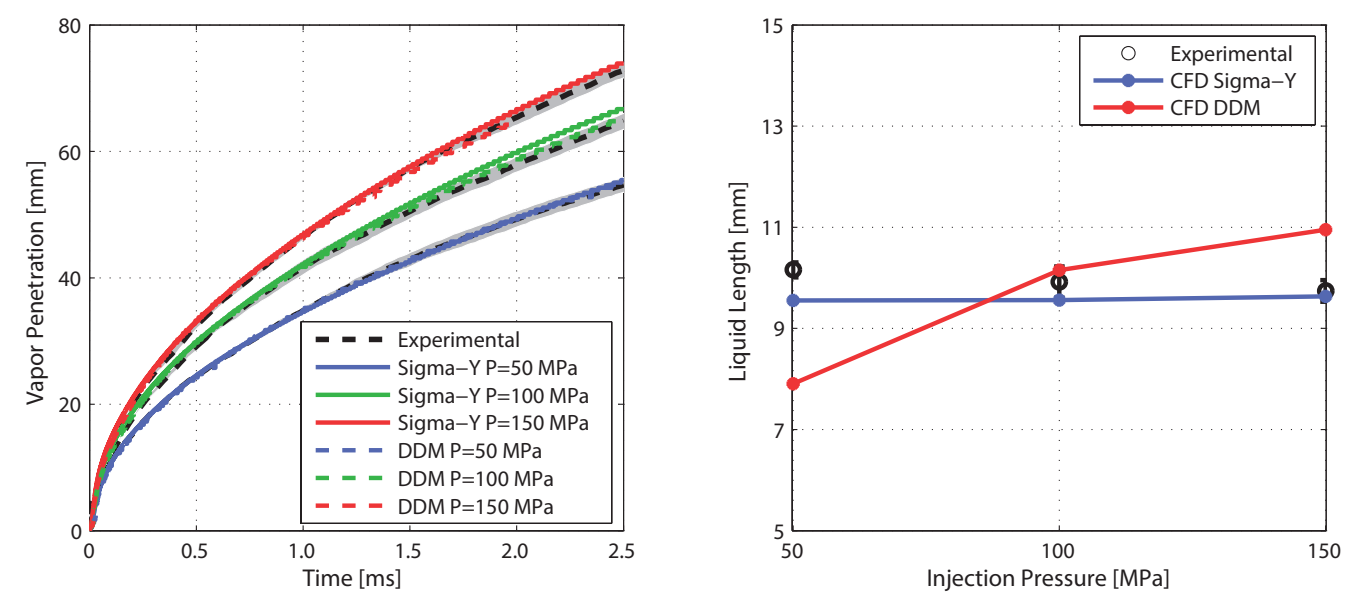

FIG. 13: Computed and measured liquid and vapor penetration for different injection pressures: Injector $210675, T_{a}=900 \mathrm{~K}$ and $\rho_{a}=22.8 \mathrm{~kg} / \mathrm{m}^{3}$. 

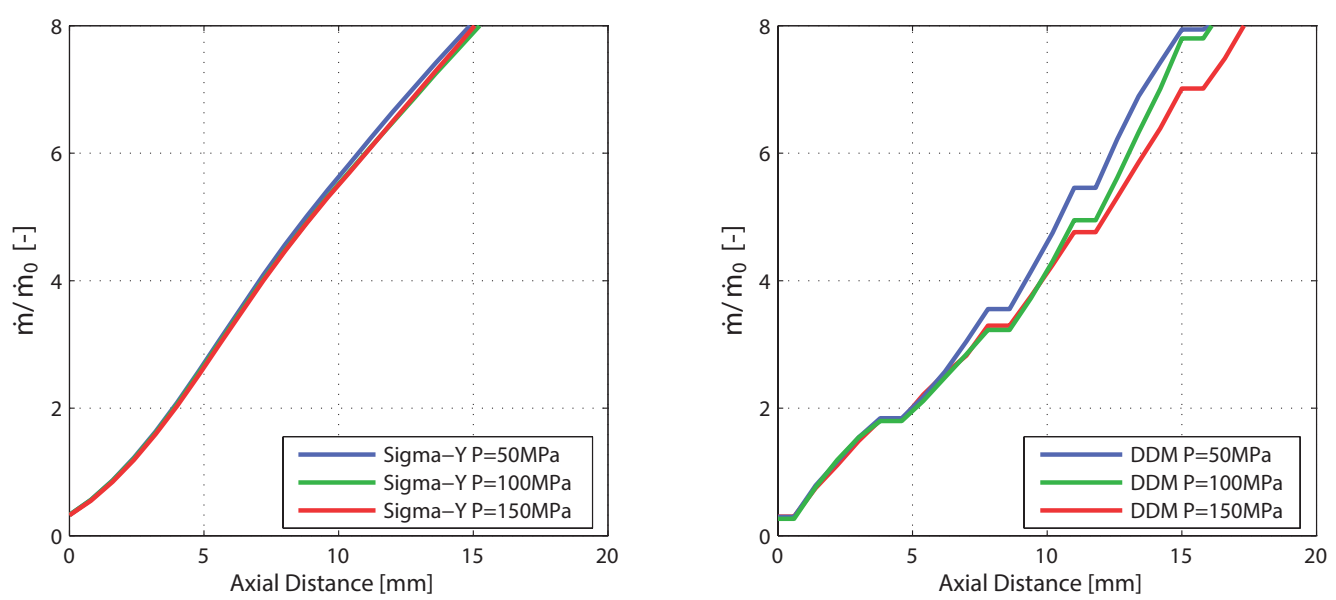

FIG. 14: (left) Entrainment comparison between $\Sigma$-Y model and (right) DDM model for different injection pressures at $2.0 \mathrm{~ms}$ after SOI: Injector $210675, T_{a}=900 \mathrm{~K}$ and $\rho_{a}$ $=22.8 \mathrm{~kg} / \mathrm{m}^{3}$.

ratios for the three different injection pressure conditions are shown, on the left for the simulations of the $\Sigma$-Y model and on the right for the DDM solver. The study is made focusing only on the first $20 \mathrm{~mm}$ of the spray, which is enough for the quasi-steady liquid length. Results for the $\Sigma$-Y model depict almost the same air entrainment, whereas the DDM model shows quite a different air entrainment among the three injection pressure conditions. It seems that droplets with lower velocity $\left(P_{\text {inj }}=50 \mathrm{MPa}\right)$, exchange momentum with the surrounding air in a noticeably faster way, which results in higher entrainment and therefore justifies the differences shown in the quasi-steady values of liquid length predictions.

\section{SUMMARY AND CONCLUSIONS}

An original OpenFOAM implementation of a Lagrangian DDM model (dieselFoam) and a fully compressible implementation of the $\Sigma$-Y model have been applied to the study of direct injection diesel sprays. Calculations have been validated against spray test rig experiments under different operating conditions using two different nozzles. Spray vapor penetration and liquid length, as well as the spatial distribution of fuel mass fraction, have been used for validation. Additionally, X-ray radiography measurements of nonevaporating Spray A conditions of ECN have been also used to evaluate the models in terms of near-field structure of diesel sprays.

The setup of both models has been carried out by comparing with Spray A baseline condition data. Good agreement with experimental measurements, including fuel mass fraction field and contours of the vapor region, were obtained by adjusting the $C_{1 \epsilon}$ constant of the $k-\epsilon$ turbulence model in the case of the $\Sigma$-Y model. On the other hand, in 
the case of DDM model, sensitivity to droplet submodels has been evaluated, in addition to the calibration of the $C_{1 \epsilon}$. The influence of these models was made explicit by means of entrainment calculations, which helps in understanding spray tip penetration trends. It has been found that $C_{1 \epsilon}$ modulates the entrainment rate, whereas CBU shifts the entrainment process spatially and B1 has almost no effect. Selected calibration values have been kept constant for all the subsequent calculations.

Predicted spray tip penetration was in quite good agreement with experimental data, although $\Sigma$-Y modeling approaches reach a better agreement in the initial zone. In addition, predicted mixture fraction profiles were slightly better in the case of $\Sigma$-Y models and specially when comparing centerline values. In the case of quasi-steady liquid lengths, both models make fairly accurate predictions, but for the different injection pressure conditions, a dependency in the Lagrangian model was noted, instead of keeping almost invariable as $\Sigma$-Y model predictions. The higher accuracy of the $\Sigma$-Y model is a consequence of both higher resolution and a more adequate modeling approach, as indicated by the noticeably better predictions of near-nozzle fuel distribution. Compared with the present contribution, analysis in the literature quite often considers only model accuracy in terms of local or penetration values. A remarkable conclusion of the present contribution is the role of entrainment calculations as an intermediate variable that links both local values and tip penetration. In summary, while a calibrated DDM model can produce very reasonable predictions in global parameters of diesel sprays, $\Sigma$-Y model performance is more accurate for representing both near nozzle and also far-field spray physics.

\section{ACKNOWLEDGMENTS}

Part of this work was possible thanks to the Programa de Ayudas de Investigación y Desarrollo (PAID-2013) of the Universitat Politècnica de València. Also,we thank the collaboration of I. García Ayala and R. Redón Moreno, students of final degree project (PFC) at the Universitat Politècnica de València.

\section{REFERENCES}

Abraham, J., What is adequate resolution in the numerical computations of transient jets?, SAE Trans. J. Engines, vol. 106, pp. 141-151, 1997.

Abraham, J. and Picket, L., Computed and measured fuel vapor distribution in a diesel spray, Atomization and Sprays, vol. 20, pp. 241-250, 2010.

Are, S., Hou, S., and Schmidt, D. P., Second order spatial accuracy in Lagrangian-Eulerian spray calculations, Numer. Heat Transfer, vol. 48, pp. 25-44, 2005.

Bardi, M., Payri, R., Malbec, L., Brunneaux, G., Pickett, L., Manin, J., Bazyn, T., and Genzale, C., Engine combustion network: Comparison of spray development, vaporization, and combustion in different combustion vessels, Atomization and Sprays, vol. 22, pp. 807-842, 2012. 
Beale, J. and Reitz, R., Modeling spray atomization with the Kelvin-Helmholtz/Rayleigh-Taylor hybrid model, Atomization and Sprays, vol. 9, no. 6, pp. 623-650, 1999.

Blokkeel, G., Barbeau, B., and Borghi, R., A 3D Eulerian model to improve the primary breakup of atomizing jet, SAE Technical Paper 2003-01-005, 2003.

Crowe, C., Schwarzkopf, J., Sommerfield, M., and Tsuji, Y., Multiphase Flows with Droplets and Particles, CRC Press, 1997.

Demoulin, F., Beau, P., Blokkeel, G., Mura, A., and Borghi, R., A new model for turbulent flows with large density fluctuations: Application to liquid atomization, Atomization and Sprays, vol. 17, pp. 315-345, 2007.

Desportes, A., Zellat, M., Desoutter, G., Liang, Y., and Ravet, F., Application of the EulerianLagrangian spray atomization (ELSA) model for the diesel injection simulation, THIESEL 2010 Conference on Thermo- and Fluid Dynamic Process in Diesel Engines, 2010.

Dukowicz, J., A particle fluid numerical model for liquid sprays, J. Comput. Phys., vol. 2, pp. 111-566, 1980.

ECN, Engine combustion network data archive, http://www.sandia.gov/ECN/, 2012

Faeth, G., Evaporation and combustion of sprays, Prog. Energy Combust. Sci., vol. 9, pp. 1-76, 1983.

García-Oliver, J., Pastor, J., Pandal, A., Trask, N., Baldwin, E., and Schmidt, D., Diesel spray CFD simulations based on the $\Sigma$-Y Eulerian atomization model, Atomization and Sprays, vol. 23, pp. 71-95, 2013.

Gorokhovski, M. and Herrmann, M., Modeling primary atomization, Ann. Rev. Fluid Mech., vol. 40, pp. 343-366, 2008.

Iyer, V. and Abraham, J., Penetration and dispersion of transient gas jets and sprays, Combust. Sci. Technol., vol. 130, pp. 315-334, 1997.

Kärrholm, F., Tao, F., and Nordin, N., Three-dimensional simulation of diesel spray ignition and flame lift-off using openfoam and KIVA-3V CFD codes, SAE Paper 2008-01-0961, 2008.

Kastengren, A., Tilocco, F. Z., Powell, C. F., Manin, J., Pickett, L. M., Payri, R., and Bazyn, T., Engine combustion network (ECN): Measurements of nozzle geometry and hydraulic behavior, Atomization and Sprays, vol. 22, pp. 1011-1052, 2012a.

Kastengren, A. L., Tilocco, F. Z., Duke, D. J., Powell, C. F., Seoksu, M., and Xusheng, Z., Timeresolved x-ray radiography of diesel injectors from the engine combustion network, ICLASS Paper, no. 1369, 2012b.

Launder, B. and Spalding, D., Mathematical Models of Turbulence, London, Academic Press, 1972.

Lebas, R., Menard, T., Beau, P., Berlemont, A., and Demoulin, F., Numerical simulation of primary break-up and atomization: DNS and modeling study, Int. J. Multiphase Flow, vol. 35, pp. 247-260, 2009.

Levich, V., Physicochemical Hydrodynamics, Prentice-Hall, 1963.

López, J. J., Novella, R., García, A., and Winklinger, J., Investigation of the ignition and combustion processes of a dual-fuel spray under diesel-like conditions using computational fluid dynamics (CFD) modeling, Math. Comput. Model., vol. 57, pp. 1897-1906, 2013. 
Lucchini, T., D’Ericco, G., and Ettorre, D., Numerical investigation of the spray-mesh-turbulence interactions for high-pressure, evaporating sprays at engine conditions, Int. J. Heat Fluid Flow, vol. 32, pp. 285-297, 2011.

Macián, V., Bermúdez, V., Payri, R., and Gimeno, J., New technique for determination of internal geometry of a diesel nozzles with the use of silicone methodology, Exp. Techniques, vol. 37, pp. 39-43, 2003.

Naber, J. and Siebers, D., Effects of gas density and vaporization on penetration and dispersion of diesel sprays, SAE Technical Paper, no. 960034, 1996.

Ning, W., Reitz, R., Diwakar, R., and Lippert, A., An Eulerian-Lagrangian spray and atomization model with improved turbulence modeling, Atomization and Sprays, vol. 19, pp. 727-739, 2009.

Pastor, J., López, J., García, J., and Pastor, J., A 1-D model for the description of mixingcontrolled inert diesel sprays, Fuel, vol. 87, pp. 2871-2885, 2008.

Pickett, L., Manin, J., Genzale, C., Siebers, D., Musculus, M., and Idicheria, C., Relationship between diesel fues spray vapor penetration/dispersion and local fuel mixture fraction, $S A E$ Int. J. Engines, vol. 4, pp. 764-799, 2011.

Pickett, L., Manin, J., Kastengren, A., and Powell, C., Comparison of near-field structure and growth of a diesel spray using light-based optical microscopy and x-ray radiography, SAE Int. J. Engines, vol. 7, no. 2, pp. 1044-1053, 2014.

Pope, S., An explanation of the turbulent round-jet/plane-jet anomaly, AIAA, vol. 16, pp. 279281, 1978.

Reid, R., Prausnitz, J., and Poling, B., The Properties of Gases and Liquids, McGraw-Hill, 1987.

Salvador, F., Gimeno, J., Pastor, J., and Martí-Aldaraví, P., Effect of turbulence model and inlet boundary condition on the diesel spray behavior simulated by an Eulerian spray atomization (ESA) model, Int. J. Multiphase Flow, vol. 65, pp. 108-116, 2014.

Schmidt, D. P. and Corradini, M. L., The internal flow of diesel fuel injector nozzles: A review, J. Engine Res., vol. 2, no. 1, pp. 1-22, 2001.

Senecal, P. K., Pomraning, E., Richards, K. J., and Som, S., Grid convergent spray models for internal combustion engines CFD simulations, Proceedings of the ASME 2012 Internal Combustion Engine Division Fall Technical Conference, no. ICEF2012-92043, 2012.

Siebers, D., Liquid-phase fuel penetration in diesel sprays, Trans. SAE, vol. 107, pp. 1205-1227, 1998.

Siebers, D., Liquid-phase fuel penetration in diesel sprays based on mixing-limited vaporization, Trans. SAE, vol. 108, pp. 703-728, 1999.

Siebers, D. L., Recent developments on diesel fuel jets under quiescent conditions, in Flow and Combustion in Reciprocating Engines, Arcoumanis, C. and Kamimoto, T. (Eds.), SpringerVerlag, Berlin, pp. 257-308, 2008.

Som, S. and Aggarwal, S., Assessment of atomization models for diesel engine simulations, Atomization and Sprays, vol. 19, pp. 885-903, 2009.

Trask, N., Schmidt, D., Lightfoot, M., and Danczyk, S., Compressible modeling of the internal flow in a gas-centered swirl-coaxial fuel injector, J. Propulsion Power, vol. 28, no. 4, pp. 685-693, 2012. 
Vallet, A. and Borghi, R., Modélisation Eulerienne de l'atomisation d'un jet liquide, C.R. Acad. Sci., vol. 327, pp. 1015-1020, 1999.

Vallet, A., Burluka, A., and Borghi, R., Development of a Eulerian model for the "atomization" of a liquid jet, Atomization and Sprays, vol. 11, pp. 619-642, 2001.

Weller, H., Tabor, G., Jasak, H., and Fureby, C., A tensorial approach to computational continuum mechanics using object-oriented techniques, Comput. Physics, vol. 12, pp. 620-631, 1998.

Xue, Q., Battistoni, M., Som, S., Quan, S., Senecal, P. K., Pomraning, E., and Schmidt, D. P., Eulerian CFD modeling of coupled nozzle flow and spray with validation against X-ray radiography data, SAE Int. J. Engines, vol. 7, no. 2, pp. 1061-1072, 2014. 